Available online on 15.9.2018 at http://ujpr.org
Universal Journal of Pharmaceutical Research
An International Peer Reviewed Journal
Open access to Pharmaceutical research

\title{
PREVALENCE OF PSEUDOMONAS AERUGINOSA (P. AERUGINOSA) AND ANTIMICROBIAL SUSCEPTIBILITY PATTERNS AT A PRIVATE HOSPITAL IN SANA'A, YEMEN \\ Ali Alyahawi ${ }^{1 *}$, Abdul Monem Alhomidi ${ }^{2}$, Nawal Al-Henhena ${ }^{3}(\mathbb{D}$ \\ ${ }^{I}$ Department of Pharmacy, Faculty of Medical Sciences, Al-Razi University, Republic of Yemen. \\ ${ }^{2}$ Faculty of Medicine, Sana'a University, Republic of Yemen. \\ ${ }^{3}$ Faculty of Laboratory Medicine, Sana'aUniversity, Republic of Yemen.
}

\section{ABSTRACT}

Objective: Pseudomonas aeruginosa is clinically significant and opportunistic pathogen that causes infections in hospitalized patients. Antibiotic resistance is a major concern in clinical practice. The ongoing emergence of resistant strains that cause nosocomial infections contributes substantially to the morbidity and mortality of hospitalized patients. Objective of present study was to estimate the prevalence of Pseudomonas aeruginosa and the antimicrobial resistance patterns of $P$. aeruginosa isolates from hospitalized patients.

Methods: The study was performed at microbiology department of a local hospital in Sana'a, Yemen. All the patients' samples of hospital departments from January, 2017 to December, 2017 were included. A Total of 2079 samples were collected during the study period. Among them, 193 strains of Pseudomonas spp. were isolated. One hundred ninety three isolates of P. aeruginosa were isolated from different clinical specimens and fully characterized by standard bacteriological procedures. Antimicrobial susceptibility pattern of each isolates was carried out by the Kirby-Bauer disk diffusion method as per CLSI guidelines. Majority of $P$. aeruginosa were isolated from Sputum, followed by urine specimens.

Results: The isolate pathogen showed the highest sensitive to Meropenem (85.5\%), followed by Amikacin (80.5\%), Imipenem (80.0\%), and Piperacillin/tazobactam (77.2). The highest frequency of resistance (96.2\%) was observed with amoxicillin /clavulinic Acid followed by cefuroxime $94.6 \%$, ampicillin/sulbactam 94.5\%, Co-Trimoxzole 80.5\%, and norfloxacin 54\%.

Conclusion: The result confirmed the occurrence of drug resistance strains of $P$. aeruginosa. Meropenem, imipenem, and amikacin, were found to be the most effective antimicrobial drugs. It therefore calls for a very judicious, appropriate treatment regimens selection by the physicians to limit the further spread of antimicrobial resistance $P$. aeruginosa.

Keywords: Antimicrobial susceptibility, Imipenem, multi drug-resistant, Pseudomonas aeruginosa.

Article Info: Received 17 July 2018; Revised 29 July; Accepted 22 August, Available online 15 September 2018 口: Cite this article-

Ali Alyahawi, Abdul Monem Alhomidi, Nawal Al-Henhena. Prevalence of Pseudomonas aeruginosa ( $P$. aeruginosa) and antimicrobial susceptibility patterns at a private hospital in Sana'a, Yemen. Universal Journal of Pharmaceutical Research 2018; 3(4): 12-18.

DOI: https://doi.org/10.22270/ujpr.v3i4.177

Address for Correspondence:

Dr. Ali Alyahawi, Department of Pharmacy, Faculty of Medical Sciences, Al-Razi University, Republic of Yemen. E-mail: alyahawipharm@yahoo.com.

\section{INTRODUCTION}

Pseudomonas aeruginosa is clinically significant and opportunistic pathogen that causes infections in hospitalized patients. In addition, most Pseudomonas species have intrinsic resistance to many antibiotics and ongoing emergence of new resistance can be developed after commonly prescribed antimicrobial agents ${ }^{1}$. Pseudomonas aeruginosa has naturally resistant to many antibiotics due to the permeability barrier afforded by its outer membrane lipopolysaccharide (LPS). Only few antibiotics are effective against Pseudomonas and even these antibiotics are not effective against all strains ${ }^{2}$. Antibiotic resistance is a major concern in clinical practice. The resistant strains of Pseudomonas aeruginosa that cause nosocomial infections contributes substantially to the morbidity and mortality of hospitalized patients ${ }^{3}$. Despite the availability of a variety of effective antimicrobial agents, treatment of pseudomonal aeruginosa is often challenging ${ }^{4}$ antimicrobial resistance is a growing problem worldwide, especially in hospitals, where resistant organisms are often first detected in ICUs ${ }^{5}$. The organism had been isolated from various infections like respiratory tract infections, cystic fibrosis, ear infections, orthopaedic infections, urinary tract infections, surgical infections, severe burns, etc. It was also reported frequently from patients undergoing 
chemotherapy for neoplastic diseases ${ }^{6}$. The variations of antibiotic protocols in clinics or in regions result in the different resistance profiles ${ }^{4}$. It is, therefore, the goal of this study to determine the prevalence of $P$. aeruginosa isolates in a private hospital in Sana'a, Yemen also to evaluate its susceptibility against certain antibiotics, as limited work has been previously conducted on this subject.

\section{METHODS}

The study was performed at university of science and technology hospital in Sana'a, Yemen. It is one of the major private hospitals in Yemen. All the patients' samples from January, 2017 to December, 2017 were included. A Total of 2079 samples were gathered during the study period. Among them, 193 strains of Pseudomonas aeruginosa were isolated. The medical records of these patients were retrieved and reviewed. All information regarding patients' gender and age as well as origin of clinical samples were collected.

Antimicrobial susceptibility testing of all the Pseudomonas aeruginosa isolates was performed by Kirby-Bauer disk diffusion method and the result were interpreted by the Clinical Laboratory Standard Institute (CLSI) guidelines ${ }^{7}$. The antimicrobial susceptibility patterns of all the Pseudomonas aeruginosa strains were determined against the following antibiotics of standard strength: ceftazidime, amikacin, gentamicin, imipenem, meropenem, ciprofloxacin, cefoperazone, piperacillin/tazobactam, amoxicillin/clavulinic acid, moxifloxacin, cefepime, ceftizoxime, ampicillin/sulbactam, cefuroxime, ceftriaxone, co-trimoxzole, and levofloxacin. Full ethical clearance was obtained from the qualified authorities who approved the study design. All data were analyzed using SPSS Statistics 21. Data was presented in tables and graphs.

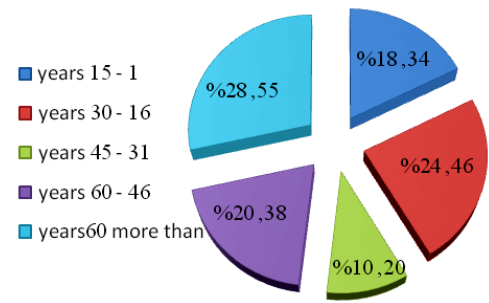

Figure 1: Distribution of $P$. aeruginosa isolates according to age.

\section{RESULTS}

According to result findings, there were more than half of Pseudomonas aeruginosa isolates in age group of 60 years and greater with $55(28 \%)$, followed by the age between 46 to 60 years in second rank about $38(20 \%)$, and finally the age between 31 to 45 years only about 20(10\%). In this study, overall Pseudomonas aeruginosa prevalence was $9.3 \%(n=193 / 2079)$. The Figure 2 showed that there were about 154(80\%) of Pseudomonas isolates form male, whereas the female had only about 39(20\%). According to the study results, the medical department had the highest prevalence of Pseudomonas aeruginosa isolates about $48(25 \%)$, followed by the intensive care unit in second rank about $41(21 \%)$, the surgical department in third rank about $37(19 \%)$ and finally the pediatric and gynecology departments had only about $16(8 \%)$.

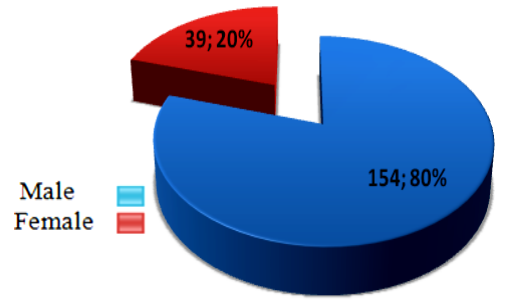

Figure 2: Distribution of $P$. aeruginosa according to gender.

The Figure 4 showed that the most of sample tests from sputum culture about $82(42.5 \%)$, followed by the sample from urine culture in second rank about $34(17.6 \%)$, and finally the sample test from other rout only about $6(3.1 \%)$.

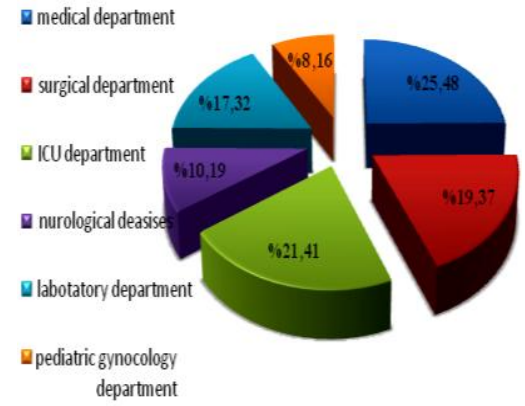

Figure 3: Distribution of $P$. aeruginosa isolates according to hospital departments.

According to the current study findings (Table 1), more than half of medication was sensitive to $P$. aeruginosa test about 12 drugs $(54.5 \%)$, whereas the medication that resistance to pseudomonas tests about 10 drugs $(45.5 \%)$.

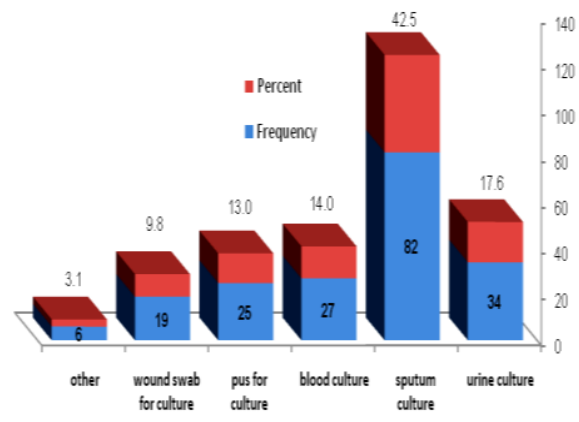

Figure 4: Distribution of P. aeruginosa isolates according to sample types.

Pseudomonas aeruginosa strains showed resistance to ciprofloxacin $50.89 \%$, ceftazidime $31.5 \%$, ceftriaxone $78 \%$, amoxicillin /clavulinic Acid 96.2\%, ampicillin/ sulbactam $94.5 \%$, cefuroxime $94.6 \%$, nalidixic acid $83 \%$, nitrofurantoin $88 \%$, doxycycline $82.6 \%$, norfloxacin $54 \%$, and Co-Trimoxzole $80.5 \%$. The highest frequency of sensitivity $(85.5 \%)$ was observed with meropenem followed by amikacin $80.5 \%$, imipenem $80 \%$, piperacilline/tazobactam $77.2 \%$, ceftizoxime $75 \%$, ciprofloxacin $71.5 \%$, levofloxacin $66 \%$, cefoperazone $64 \%$, gentamicin $56 \%$, ceftazidime $54.5 \%$, moxifloxacin 49\%, and cefepime $44.5 \%$. According to Figure 5 below, the highest resistance rate of anti-pseudomonal agent was with cefepime about $43.5 \%$ and the lowest resistance rate with 
imipenem. Resistance to antipseudomonal drugs in current study was found to be cefepime (43.5\%), ceftazidime $(31.5 \%)$, ciprofloxacin $(24 \%)$, piperacillin /tazobactam (16.5\%), imipenim (15.4\%). In the present study, multi drug resistance (MDR) rate (resistance to three or more of anti-Pseudomonal antimicrobials (i.e. piperacillin+tazobactam, imipenem, ceftazidime and amikacin) was determined to be $4.2 \%(8 / 193)$. Also MDR rate for only three anti Pseudomonal antimicrobials without imipenem was $4.2 \%(n=8 / 193)$ (i.e. piperacillin+tazobactam, ceftazidime and amikacin)

Table 1: Antimicrobial susceptibility patterns for $P$. aeruginosa isolates

\begin{tabular}{|c|c|c|c|c|c|c|c|}
\hline \multirow{2}{*}{ Antibiotics } & \multirow{2}{*}{$\begin{array}{l}\text { Expected } \\
\text { options }\end{array}$} & \multicolumn{2}{|c|}{ Response } & \multirow[t]{2}{*}{ Antibiotics } & \multirow{2}{*}{$\begin{array}{l}\text { Expected } \\
\text { options }\end{array}$} & \multicolumn{2}{|c|}{ Response } \\
\hline & & $\mathbf{F}$ & $\%$ & & & $\mathbf{F}$ & $\%$ \\
\hline \multirow{3}{*}{ Ceftriaxone } & $\mathrm{S}$ & 11 & 18.5 & \multirow{3}{*}{ Ceftazidime } & $\mathrm{S}$ & 103 & 54.5 \\
\hline & $\mathrm{R}$ & 46 & 78 & & $\mathrm{R}$ & 60 & 31.5 \\
\hline & I & 2 & 3.5 & & $\mathrm{I}$ & 26 & 14 \\
\hline \multirow{3}{*}{$\begin{array}{l}\text { Cefoperazone } \\
\text { sulbactam }\end{array}$} & $S$ & 58 & 64 & \multirow{3}{*}{ Ciprofloxacin } & $S$ & 118 & 71.5 \\
\hline & $\mathrm{R}$ & 27 & 29.5 & & $\mathrm{R}$ & 40 & 24 \\
\hline & I & 6 & 6.5 & & I & 7 & 4.5 \\
\hline \multirow{3}{*}{ Levofloxacin } & $S$ & 108 & 66 & \multirow{3}{*}{$\begin{array}{l}\text { Co- } \\
\text { Trimoxzole }\end{array}$} & $S$ & 37 & 19.5 \\
\hline & $\mathrm{R}$ & 44 & 26.8 & & $\mathrm{R}$ & 152 & 80.5 \\
\hline & $\mathrm{I}$ & 12 & 7.2 & & $\mathrm{I}$ & 0 & 0.0 \\
\hline \multirow{3}{*}{$\begin{array}{l}\text { Ampicillin/ } \\
\text { sulbactam }\end{array}$} & $S$ & 2 & 3.7 & \multirow{3}{*}{ Imipenem } & $\mathrm{S}$ & 150 & 80 \\
\hline & $\mathrm{R}$ & 51 & 94.5 & & $\mathrm{R}$ & 29 & 15.4 \\
\hline & I & 1 & 1.8 & & I & 9 & 4.6 \\
\hline Amoxicillin / & $S$ & 4 & 2.1 & \multirow{3}{*}{ Norfloxacin } & $S$ & 10 & 38.5 \\
\hline Clavulinic & $\mathrm{R}$ & 179 & 96.2 & & $\mathrm{R}$ & 14 & 54 \\
\hline Acid & I & 3 & 1.7 & & I & 2 & 8 \\
\hline \multirow{3}{*}{ Amikacin } & $\mathrm{S}$ & 152 & 80.5 & \multirow{3}{*}{ Cefepime } & $\mathrm{S}$ & 83 & 44.5 \\
\hline & $\mathrm{R}$ & 28 & 14.8 & & $\mathrm{R}$ & 81 & 43.5 \\
\hline & I & 9 & 4.7 & & I & 22 & 12 \\
\hline \multirow{3}{*}{ Gentamicin } & $S$ & 105 & 56 & \multirow{3}{*}{ Meropenem } & $S$ & 89 & 85.5 \\
\hline & $\mathrm{R}$ & 65 & 35 & & $\mathrm{R}$ & 10 & 9.5 \\
\hline & $\mathrm{I}$ & 17 & 9 & & $\mathrm{I}$ & 5 & 5 \\
\hline \multirow{3}{*}{ Moxifloxacin } & $S$ & 77 & 49 & \multirow{3}{*}{$\begin{array}{l}\text { Piperacillin/ } \\
\text { tazobactam }\end{array}$} & $S$ & 146 & 77.2 \\
\hline & $\mathrm{R}$ & 69 & 44 & & $\mathrm{R}$ & 31 & 16.5 \\
\hline & I & 11 & 7 & & $\mathrm{I}$ & 12 & 6.3 \\
\hline \multirow{3}{*}{ Cefuroxime } & $S$ & 8 & 4.2 & \multirow{3}{*}{ Ceftizoxime } & 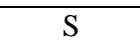 & 1 & 4 \\
\hline & $\mathrm{R}$ & 178 & 94.6 & & $\mathrm{R}$ & 18 & 75 \\
\hline & I & 2 & 1.2 & & I & 5 & 21 \\
\hline
\end{tabular}

\section{DISCUSSION}

Pseudomonas aeruginosa has defined as one of the most common nosocomial pathogens. Hence we have undertaken this study to analyze the prevalence and antimicrobial susceptibility pattern of Pseudomonas aeruginosa from various clinical samples of a private hospital. Periodic antimicrobial resistance monitoring in $P$. aeruginosa is fundamental to updating the current activity level of commonly used antipseudomonal drugs. The present study measures the rate of isolation of Pseudomonas aeruginosa $(\mathrm{n}=193 / 2079 ; 9.3 \%)$ as which is lower than previous studies as by Tadvi et $a l .{ }^{8}(22.67 \%)$ and Viren et $a l^{9}$. The occurrence of $P$. aeruginosa was found to be higher in males, inpatients in age group >60 years and in surgery department, which is same as reported by Marzoqi et al., ${ }^{10}$. This might be due to prolonged hospitalization and other associated co-morbidities in these age groups. The distribution of Pseudomonas aeruginosa isolates specimens may vary with each hospital as each hospital and each health facility has a different environment associated with it. According to the study results, more

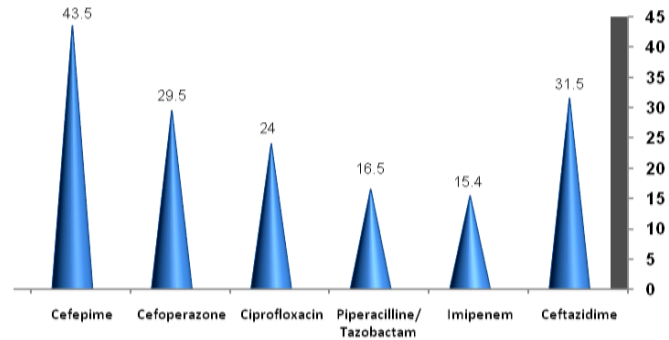

Figure 5: Resistance rates of anti-pseudomonal agent. than $42.5 \%$ of the Pseudomonas aeruginosa isolates were obtained from sputum samples. The distribution rank of the isolates according to the types of specimens was (respiratory sputum $>$ urine $>$ blood $>$ pus $>$ wound swap > others). Respiratory isolates $(42.5 \%)$ were the most frequently encountered. $P$. aeruginosa isolates from respiratory tract as observed in a similar study of inpatient isolates done in a Saudi Arabian hospital ${ }^{11}$. In the present study, the maximum clinical isolates of $P$. aeruginosa were isolated from medical department (25\%), followed by ICU $(21 \%)$ and surgical department (19\%). This was similar to study of Pathmanathan $\mathrm{SG}^{12}$. The distribution of specimens of Pseudomonas aeruginosa might vary with each hospital as each hospital facility has a different environment associated with it. The correlation between specimen type and multidrug resistance would have been more noteworthy if supported by data on patients' clinical conditions. Prevalence of infection was higher in medical ward followed by ICU as maximum isolates were isolated from sputum samples. 
There was statistical significant relationship between the piperacilline/tazobactam susceptibility and sample types $(\mathrm{P}$ value $=0.04)$. On other hand, there was no statistical significant relationship between the other antibiotics susceptibility (ceftazidime, imipenem, cefipeme) and sample types. As with this study, $P$. aeruginosa infection was primarily noted among older adults $(\mathrm{n}=55,28 \%)$ particularly respiratory infection ( $\mathrm{n}$ $=82,42.5 \%)$. There are a number of reasons why older adults are burdened by this type of infection. These include age-associated impairments in immunity that lead to reduced response to vaccination, a constellation of chronic and comorbid diseases, and functional limitations associated with advanced age. Additionally, older adults are at risk for aspiration pneumonia, outbreaks of gastroenteritis, recurrent urinary tract infection, and prosthetic device infections ${ }^{13}$. In the European Prevalence of Infection in Intensive Care (EPIC), P. aeruginosa was predominant gram-negative bacteria isolated from broncho pulmonary infections and accounts for $17 \%$ of health care-associated pneumonia and late-onset ventilate associated pneumonia $^{14}$ and accounts for significant cases of cystic fibriosis ${ }^{15}$.The distribution of isolates differs with studies and clinical specimens ${ }^{16}$. Intensive care patients especially create an environment for infection because of the debilitating effect of a prolonged hospitalisation and the application of medical equipment (airways, catheters etc) ${ }^{17}$. ICUs are generally considered epicenters of antibiotic resistance and the principal sources of outbreaks of multi-resistant bacteria. The most important risk factors are excessive consumption of antibiotics exerting selective pressure on bacteria, the frequent use of invasive devices and relative density of a susceptible patient population with severe compelling diseases ${ }^{18}$. Thus, in ICUs, empirical antibiotic treatments should be avoided and treatment should be carried out using antibiotic susceptibility tests. ICUs should be regularly monitored resistance pattern against the various antibiotics. P. aeruginosa was responsible for pneumonia and septicaemia with deaths rate about $30 \%$ in immune compromised patients ${ }^{19 .}$ In the current study results, Pseudomonas aeruginosa showed resistance to amoxicillin/ clavulinic Acid $96.2 \%$, ampicillin/ sulbactam $94.5 \%$, cefuroxime $94.6 \%$, nalidixic acid $83 \%$, nitrofurantoin $88 \%$, doxycycline $82.6 \%$, ciprofloxacin $50.89 \%$, ceftazidime $31.5 \%$, ceftriaxone $78 \%$, norfloxacin $54 \%$, and cotrimoxzole $80.5 \%$. However, the highest frequency of sensitivity $(85.5 \%)$ was observed with meropenem followed by amikacin 80.5\%, imipenem $80 \%$, piperacilline/tazobactam $77.2 \%$, ceftizoxime $75 \%$, ciprofloxacin $71.5 \%$, Levofloxacin $66 \%$, Cefoperazone $64 \%$, Gentamicin 56\%, ceftazidime $54.5 \%$, moxifloxacin $49 \%$, and cefepime $44.5 \%$. This may be explained by the fact that routine use of these antibiotics can lead to clinically significant resistance. One remarkable finding in the present study was the highest frequency of sensitivity $(85.5 \%)$ was observed with meropenem, $85.5 \%$, amikacin $(80.5 \%)$, and piperacillin/ tazobactem (77.2\%). These drugs were the most effective drugs against $P$. aeruginosa infections. This similar to study finding by Taranasarwat et al., ${ }^{20}$, who reported highest sensitivity to imipenam. Also it was quite similar to the findings of Shaikh et al., $(100 \%)^{21}$ and Mohan et al., $(94.3 \%)^{22}$. One striking feature in this study was that all the $P$. aeruginosa isolates were found to be sensitive to imipenem. This may be due to the restricted use of imipenem in this hospital. This is consistent with a report published in 2002 in Mangalore, India ${ }^{23}$. The emergence of carbapenem resistance is a serious concern ${ }^{24}$. In various studies across the world, varying rates of resistance from $4-60 \%$ have been reported for imipenem and meropenem ${ }^{25}$. Another survey found that resistance to imipenem was $19 \%$, while other studies have reported low rates $(5.8 \%$ and $9 \%$ ) and high rates $(38.6 \%)$ of resistance to imipenem ${ }^{26}$. Piperacillin+ tazobactam showed a sensitive rate of $77.2 \%$ in this study and cefoperazone-sulbactum showed a lower resistance of $29.5 \%$ only, indicating beta-lactamase inhibitor markedly expands the spectrum of activity of betalactams, which makes the combination drug the preferred choice against Pseudomonas aeruginosa infections. Thus, emphasis should be given towards use of combined antibiotics in the treatment of pseudomonal infections ${ }^{27}$. Bayani et al., found that the resistance rate of $P$. aeruginosa to amikacin, ceftazidime, cefepime, imipenem, and ciprofloxacin was $53.3 \%, 43.3 \%, 40 \%, 40 \%$, and $33.3 \%$, respectively, and the prevalence of $P$. aeruginosa resistant isolates has increased ${ }^{28}$. According to previous evidence, the rate of susceptibility was most productive for antimicrobial agent of class carbapenem against Pseudomonas aeruginosa ${ }^{29}$. Supported current results as $85.5 \%$ of strains were susceptible to Meronem and $80 \%$ to imipenem of class carbapenems. Although the resistance to carbapenems that include imipenem (16\%) and meropenem (17.1) was low in this study, quite alarming should take into account that carbapenems are the last line of antibiotics for treating Gram-negative bacilli infections. Resistance to carbapenems may be due to a result of complex interactions of several mechanisms including production of carbapenemase, overproduction of efflux system and loss of outer membrane porins. $P$. aeruginosa isolates that are carbapenem resistant, specifically carbapenemase producing, are the worst, for the reason that they are associated with a higher mortality rate ${ }^{24}$. Amikacin in this study was noted to be the most effective drug ( $80.5 \%$ sensitive). However, it is not commonly prescribed drug, because of its numerous side effects including renal toxicity, blurred vision, hearing loss, Bartter-like syndromes ${ }^{30}$, neuromuscular blockade, arthralgia, and apnoea. In addition, ciprofloxacin $(71.5 \%$ sensitive $)$ proved to be within the most effective drugs for routine use among the $P$. aeruginosa strains investigated in this study. The result finding in this study was similar in a previous study finding that reported that amikacin had the highest sensitivity against $P$. aeruginos ${ }^{9}$. Also in France, a higher susceptibility rate of $86 \%$ of amikacin was reported by Cavallo et al., ${ }^{31}$. An earlier study reported from Kathmandu, Nepal ${ }^{32}$ shown amikacin (81.4\% sensitive) and ciprofloxacin (70.3\% sensitive) among $P$. aeruginosa strains examined. Amikacin 
seems to be a promising therapy for pseudomonal infection. Hence, its use should be restricted to severe nosocomial infections, in order to avoid rapid emergence of resistant strains $^{33}$. However, high resistance to aminoglycosides had been reported in studies done in Bangladesh ${ }^{34}$, Turkey ${ }^{4}$ and Malaysia ${ }^{35}$. Similarly, higher rates of resistance to fluoroquinolones such as ciprofloxacin resistance $(92 \%)$ were shown in a study from Malaysia ${ }^{36}$. Also study findings by Zhanel et al., reported moxifloxacin $58 \%$ and ciprofloxacin $46.7 \%^{37}$. Because of the increasing resistance to fluroquinolone in many hospitals, its empirical usage is either banned or restricted, to bring the developing resistance rates under control. Recently, ceftazidime and cefepime are the most frequently prescribed third and fourth generation cephalosporins respectively. Ceftadizime is known antipseudomonal drug that has demonstrated high susceptibility pattern with $P$. aeruginosa isolates. The increased prevalence of ceftazidime resistant $P$. aeruginosa is related to the increased use of beta lactam antibiotics such as amoxicillin and ceftazidime. However, the resistance to cefadizime was reported as $31.5 \%$ in this study. This value of resistance was less than reported from Gujarat, with a resistance value of $75 \%{ }^{9} . P$. aeruginosa strains in this study exhibited a high rate of resistance to the third generation cephalosporin drug such as ceftriaxone (78\%). A much higher resistance to ceftriaxone of $75 \%, 86 \%$ and $93.9 \%$ had been reported in studies done in India ${ }^{38}$ Bangladesh $^{34}$ and $\mathrm{Nepal}^{27}$. Several studies have confirmed that Pseudomonas aeruginosa was mostly resistant against ceftriaxone. However, this high level of resistance is not quite surprising as some suggest that ceftriaxone has considerably low activity against $P$. aeruginosa ${ }^{39,40}$. Another study reported the following rates of resistance to cefepime $64.8 \%$, piperacilline/tazobatctam $45 \%$, ciprofloxacin $38.9 \%$, levofloxacin $36.1 \%$, gentamicin $37.3 \%$ and amikacin $30 \%{ }^{41}$. Relatively low piperacilline/tazobatctam resistance $(11.5 \%)$ had been reported in a hospital isolates of $P$. aeruginosain a study from Saudi Arabia $^{11}$. In a study done in Kathmandu, Nepal ${ }^{27}, P$. aeruginosa isolates obtained from intensive care unit of a national heart centre showed a high cefoperazonesulbactum sensitivity rate of $84.8 \%$. A previous study discovered an increased mortality rate associated with empiric piperacillin-tazobactam therapy given to patients with $P$. aeruginosa bacteraemia; the isolates had reduced piperacillin-tazobactam susceptibility ${ }^{42}$. In this study, amoxicillin /clavulinicacid had established $96.2 \%$ resistance. Similarly, in a study conducted in Pakistan reported by Khan et al., ${ }^{43}$ had a high resistance rate of penicillin that is $98 \%$; current findings are also in agreement with other studies as reported by Sasirekha et al., ${ }^{44}$ and Ullah et al., ${ }^{45}$ with respect to penicillin's. Also the same findings were obtained with amoxicillin/clavulinic acid (1.88\%) and showed increasing resistance. Multi drug efflux pumps in the inner and outer membrane of Ps. Aeruginosa may protect the bacterium from $\beta$-lactam agents ${ }^{46}$. Similar pattern had been reported in study in Nigeria ${ }^{47}$. In addition, susceptibility to fourth-generation such as cefepime reported in India $32 \%$ and in Bulgaria
$42 \%$ against Pseudomonas aeruginosa isolates. The high resistance to cephalosporins may be due to production of extended spectrum $\beta$-lactamases by the bacteria involved $^{50}$. Cefuroxime was one of the cephalosporin drugs tested in this study, with resistance value of $94.6 \%$. This high resistance value observed were comparable with the report from Gujarat, India with resistance value of $73.2 \%$, but higher than reports from Malaysia of $40 \%$. Selective pressure from the use of antimicrobial agents is a major determinant for the emergence of resistant strains. The rate of resistance for the anti-folate drug co-trimoxazole in the present study was $80.5 \%$. In similar to previous study done in Bangladesh ${ }^{34}$ showed rate of resistance for cotrimoxazole to be $93.5 \%$ in wound swab and pus isolates of $P$. aeruginosa while a Nigerian study ${ }^{52}$ showed $P$. aeruginosa isolates $100 \%$ resistant to cotrimoxazole. According to resistance rates of antipseudomonal agents, imipenem and piperacillin /tazobactam were found to be effective when compared to Ceftazidime, cefepime, and Ciprofloxacin. So, imipenem which is both an anti-pseudomonal drug and carbapenem was the best drug. According to the study findings, MDR rate (resistance to three or more of anti Pseudomonal antimicrobials (i.e. piperacillin+ tazobactam, imipenem, ceftazidime and amikacin) was determined to be $4.2 \%$ (8/193). Also MDR rate for only three anti Pseudomonal antimicrobials without imipenem was $4.2 \% \quad(8 / 193)$ (i.e. piperacillin+ tazobactam, ceftazidime and amikacin). A study done by Unan et al., ${ }^{53}$. in Turkey reported rates of MDR, which were as high as $60 \%$, whereas study done by Sabir et al., in Pakistan detected lower rates of MDR $(22.08 \%)^{54}$. Moreover, the rate of current study was lower than a study done in Egypt, where Gad et al., ${ }^{\mathbf{5 5}}$ observed 36\% MDR P. aeruginosa. On comparing the sensitivity patterns of these antimicrobials, it was found that there was a considerable difference in the sensitivity pattern among these studies. This indicates that the sensitivity pattern changes from hospital to hospital and population to population. This has been possibly resulted from indiscriminate use of antibiotic, lack of awareness, patient non-compliance and unhygienic conditions ${ }^{56}$. According to Berglund ${ }^{57}$ one of the reasons for resistance among bacteria is a result of either overuse and misuse of antibiotics. By misuse, this refers to the prescription of antibiotics without establishing bacterial infection, and the noncompliance of the patient to the full prescription. Moreover, antibiotic resistance can also be transferred horizontally between bacteria. The current study results indicated that $P$. aeruginosa was becoming resistant to commonly used antibiotics due to excessive consumption. The empirical antibiotic treatment should be limited and treatment should be carried out using antibiotic susceptibility test and efforts should be made to prevent spread of resistant bacteria ${ }^{56}$.

\section{CONCLUSION}

In conclusion, results of the present study clearly demonstrated the occurrence of resistance to various antipseudomonal agents among the Pseudomonas aeruginosa isolates. The statistics in this study showed 
low rates of antibiotic resistance to meropenem, amikacin, and meropenem, and piperacillin/ tazobactam and maximum sensitivity against Pseudomonas aeruginosa strains. We suggest a more restricted and a more rational use of these drugs in hospital setting in order to avoid rapid emergence of resistant strains. Regular anti-microbial susceptibility monitoring is essential of local, regional and national level isolates. Every effort should be made to prevent spread of resistant organisms. The solution can be planned by continuous efforts of microbiologist, clinician, pharmacist and community to promote greater understanding of this problem. Frequent hand washing to prevent spread of organism should be encouraged.

\section{AUTHOR'S CONTRIBUTION}

The manuscript was carried out, written, and approved in collaboration with all authors.

\section{ACKNOWLEDGEMENTS}

The authors extend their thanks and appreciation to the Al-Razi University, Republic of Yemen to provide necessary facilities for this work.

\section{CONFLICT OF INTEREST}

The authors declare that they have no competing interests.

\section{REFERENCES}

1. Kenneth T. Textbook of bacteriology: Pseudomonas aeruginosa. Wisconsin University, France 2004; 7-15.

2. ALshaiki JMM, Toweir AA. Prevalance Pseudomonas aeruginosa Among Libyan Patients and its Association with Hospital's Environment in Benghazi. J Med Microb Diagn 2017; 6: 257.https://doi.org/10.4172/2161-0703.1000257

3. Acar JF. Consequences of bacterial resistance to antibiotics in medical practice. Clin Infect Dis 1997; 24 (1):17 8. https://doi.org/10.1093/clinids/24.Supplement_1.S17

4. Savas L, Duran N, Savas N, Onlen Y, Ocak S. The prevalence ans resistance patterns of Pseudomonas aeruginosa in intensive care units in a university hospital. Turk J Med Sci 2005; 35:317-22.

5. Carmeli Y, Troillet N, Eliopoulos GM, Samore MH. Emergence of antibiotic-resistant Pseudomonas aeruginosa: comparison of risks associated with different anti pseudomonal agents. Antimicrob Agents Chemother 1999; 43: 1379-82. PMID: 10348756

6. Renuga S, Lakshmi K, Chitralekha S, Illamani V. Prevalence of Pseudomonas aeruginosa and its antibiotic susceptibility pattern in a Tertiary Care Hospital. Int J Res Pharm Sci 2015; 6(1), 27-30.

7. Cockerill FR (Ed.), Performance Standards for Antimicrobial Susceptibility Testing: Twenty-first Informational Supplement, Clinical and Laboratory Standards Institute (CLSI), 2011.

8. Tadvi J, Javadekar TB, Bhavsar R, Garala N. Prevalence and antibiogram of Pseudomonas aeruginosa at S.S.G. Hospital, Baroda, Gujarat, India. J Res Med Den Sci 2015; 3:204-207. https://doi.org/10.5455/jrmds.20153310

9. Javiya VA, Ghatak SB, Patel KR, Patel JA. Antibiotic susceptibility patterns of Pseudomonas aeruginosa at a tertiary care hospital in Gujarat, India. Indian J Pharmacol. 2008; 40:230-4. https://doi.org/10.4103/0253-7613.44156

10. Al-Marzoqi AH, Al Taee ZM. Pseudomonas aeruginosa: Antibiotic resistance pattern to different isolates in Al-Hillah city, Iraq. J Nat Sci Res 2013; 3:23-30.

https://doi.org/10.3389/fmicb.2016.00586
11. Al-Tawfiq JA. Occurrence and antimicrobial resistance pattern of inpatient and outpatient isolates of Pseudomonas aeruginosa in a Saudi Arabian hospital: 19982003. Int J Infect Dis 2007; 11:109-114. https://doi.org/10.1016/j.ijid.2005.11.004

12. Pathmanathan SG, Samat NA, Mohamed R. Antimicrobial susceptibility of clinical isolates of Pseudomonas aeruginosa from a Malaysian Hospital. The Malaysian J Med Sci 2009; 16(2):27-32. PMID: 22589655

13. Tortora G, Funke B, Case C. Microbiology: An Introduction, $6^{\text {th }}$ ed.; Benjamin Cummings: California, CA, USA, 1998.

14. Vincent JL, Bihari DL, Suter PM, Bruining HA, White J, Nicolas-chanion $\mathrm{M}$. The prevalence of nosocomial infection in intensive care units in Europe: results of the European Prevalence of Infection in Intensive Care (EPIC) study. J American Med Asoc 1995; 74:639-644. PMID: 7637145

15. Pier GB. Role of cystic fibrosis transmembrane conductance regulator in innate immunity to Pseudomonas aeruginosa infections. Proceedings of National Academy of Science, USA. 2000; 97:8822-8828. https://doi.org/10.1073/pnas.97.16.8822

16. Okon KO, Agukwe PC, Oladosu W, Balogun ST, Uba A. Antibiotic resistance pattern of Pseudomonas aeruginosa isolated from clinical specimens in a tertiary care hospital in Northeastren Nigeria. Internet J Microbiol 2010;8:1-6 https://doi.org/10.4103/ijmr.IJMR_14_18

17. Jarlier V, Fosse T, Philippon A. Antibiotic susceptibility in aerobic gram-negative bacilli isolated in intensive care units in 39 French teaching hospitals (ICU study). Intensive Care Med 1996; 22: 1057-65. https://doi.org/10.1086/647284

18. Weber DJ, Raasch R, Rutala WA. Nosocomial infections in the ICU: the growing importance of antibiotic-resistant pathogens. Chest 1999; 115: 34S-41S. https://doi.org/10.1378/chest.115.suppl_1.34S

19. Olayinka AT, Onile BA, Olayinka BO. Prevalence of multidrug resistant (MDR) Pseudomonas aeruginosa isolates in Surgical Units of Ahmadu Bello University Teaching Hospital, Zaria, Nigeria: an Indication for Effective Control Measures Annals of African Medicine. 2004; 3 (1):13-16.

20. Sarwat T, Rashid M, RastogiV, Chander Y. A comparative study of Antibiogram of Pseudomonas aeruginosa in Hospital and community acquired infections. Int J Curr Microbiol App Sci 2015; Special Issue-1: 286-291 https://doi.org/10.1016/j.sjbs.2014.06.0

21. Shaikh S, Fatima J, Shakil S, Mohd S, Rizvi D, Kamal MA. Prevalence of multidrug resistant and extended spectrum beta-lactamase producing Pseudomonas aeruginosa in a tertiary care hospital. Saudi J Biol Sci 2015; 22, 62-64 https://doi.org/10.1289/EHP292

22. Mohan BS, Lava R, Prashanth HV, Nambiar V, Metri B, Nayak V, Sri Krishna R. Prevalence and antibiotic sensitivity pattern of Pseudomonas aeruginosa; an emerging nosocomial pathogen. Int J Biol Med Res 2013; 4(1): 27292731.https://doi.org/10.1155/2011/605195

23. Shenoy S, Baliga S, Saldanha DR, Prashanth HV. Antibiotic sensitivity patterns of Pseudomonas aeruginosa strains isolated from various clinical specimens. Indian J Med Sci 2002; 56(9):427-30. https://doi.org/10.4103/02537613.44156

24. Liu Q, Li X, Li W. Influence of carbapenem resistance on mortality of patients with Pseudomonas aeruginosa infection: a meta-analysis. Sci Rep. 2015; 5, 11715. https://doi.org/10.1038/srep11715

25. Gonlugur U, Bakici MZ, Akkurt I, Efeoglu T. Antibiotic susceptibility patterns among respiratory isolates of Gramnegative bacilli in a Turkish University hospital. BMC Microbiol 2004; 4: 32. https://doi.org/10.1186/1471-2180-432

26. Khan M.A., Faiz A. Antimicrobial resistance patterns of Pseudomonas aeruginosa in tertiary care hospitals of Makkah and Jeddah. Ann Saudi Med 2016; 36: 23-28. https://doi.org/10.5144/0256-4947.2016.23 
27. Bhandari S, Banjara MR, Lekhak B, Bhatta DR, Regmi SR. Multi-drug and pan-drug resistant Pseudomonas aeruginosa: a challenge in post-antibiotic era. Nepal J Sci Tech 2012; 13(2):197-202. https://doi.org/10.3126/njst.v13i2.7736

28. Bayani M, Siadati S, Rajabnia R, Taher AA. Drug Resistance of Pseudomonas aeruginosa and Enterobacter cloacae Isolated from ICU, Babol. Northern Iran Int J Mol Cell Med 2012; 2: 204-209. PMID: 24551814

29. Turner PJ. Meropenem and imipenem activity against Pseudomonas aeruginosa isolates from the MYSTIC Program, Diagnostic Microbiology and Infectious Disease 2006; 56 (3): 341-344. https://doi.org/10.1016/j.diagmicrobio.2006.07.015

30. Juayang AC, Maestral DG Jr, Gallega CT, et al. Review on the antimicrobial resistance of pathogens from tracheal and endotracheal aspirates of patients with clinical manifestations of pneumonia in Bacolod City in 2013. Int $\mathrm{J}$ Bacteriol 2015; 2015:942509.https://doi.org/10.1155/2015/942509

31. Cavallo JD, Hocquet D, Plesiat P, Fabre R, RousselDelvallez M. Susceptibility of Pseudomonas aeruginosa to antimicrobials: a 2004 French multicentre hospital study. J Antimicr Chemo 2007; 59, (5): 1021-1024. https://doi.org/10.1093/jac/dkm076

32. Koirala P, Bhatta DR, Ghimire P, Pokhrel BM, Devkota U. Bacteriological profile of tracheal aspirates of the patients attending a neuro-hospital of Nepal. Int J Life Sci 2010;4:6065. https://doi.org/10.1155/2013/847569

33. Poole K. Aminoglycosides resistance in Pseudomonas aeruginosa. Antimicrob Agents Chem 2005; 49:479-87. https://doi.org/10.1128/AAC.49.2.479-487.2005

34. Rashid A, Chowdhury A, Rahman SHZ, Begum SA, Muazzam N. Infections by Pseudomonas aeruginosa and antibiotic resistance pattern of the isolates from Dhaka Medical College Hospital. Bangladesh J Med Microbiol 2007; 1(2):48-51.https://doi.org/10.1186/s13104-015-1497-x

35. Fazlul MKK, Zaini MZ, Rashid MA, Nazmul MHM. Antibiotic susceptibility profile $\mathrm{s}$ of clinical isolates of Pseudomonas aeruginosa from Selayang Hospital, Malaysia. Biomed Res 2011; 22(3):263-66. PMID: 22589655

36. Al-KabsiAM, Yusof MYBM, Sekaran SD. Antimicrobial resistance pattern of clinical isolates of Pseudomonas aeruginosa in the University of Malaya Medical Center, Malaysia. Afr J Microbiol Res 2011; 5(29):5266-72. https://doi.org/10.5897/AJMR11.284

37. ZhanelG G, LaingN M, Nichol K. A. Antibiotic activity against Urinary Tract Infection (UTI) isolates of Vancomycin Resistant Enterococci (VRE): results from the 2002 North American vancomycin resistant enterococci susceptibility study (NAVRESS). J Antim Chem 2003; 52 (3): 382-388. https://doi.org/10.1093/jac/dkg352

38. Arora D, Jindal N, Kumar R, Romit. Emerging antibiotic resistance in Pseudomonas aeruginosa. Int $\mathrm{J}$ Pharm Pharm Sci 2011; 3(2):82-4 https://doi.org/10.12688/f1000research.19509.1

39. Bassetti D, Cruciani M, Solbiati M, Rubini F, Gandola L, Valenti G, et al. Comparative efficacy of ceftriaxone versus ceftazidime in the treatment of nosocomial lower respiratory tract infections. Chemotherap 1991; 37:371-5. 20. https://doi.org/10.1128/JCM.00893-06

40. Mody L, Bradley SF, Strausbaugh LJ, Muder RR. Prevalence of ceftriaxone and ceftazidime resistant gram-negative bacteria in long term-care facilities. Infect Control Hosp Epidemiol 2001; 22(4):193-4.https://doi.org/10.1086/503397

41. Dash M, Padhi S, Narasimham MV, Pattnaik S. Antimicrobial resistance pattern of Pseudomonas aeruginosa isolated from various clinical samples in a tertiary care hospital, South Odisha, India. Saudi J Health Sci 2014; 3, 15 19. https://doi.org/10.4103/2278-0521.130200

42. Tam VH, Gamez EA, Weston JS, Gerard LN, LaRocco MT, Caeiro JP, et al. Outcomes of Bacteremia due to Pseudomonas aeruginosa with Reduced Susceptibility to
Piperacillin-Tazobactam: Implications on the appropriateness of the resistance breakpoint. Clin Infect Dis 2008; 46:862867. https://doi.org/10.1086/528712

43. Khan JA, Iqbal Z, Ur Rahman S, Farzana K, Khan A. Prevalence and resistance pattern of Pseudomonas aeruginosa against various antibiotics. Pakistan J Pharm Sci.2008; 21(3):311-315.

44. Sasirekha B, Manasa R, Ramya P, Sneha R. Frequency and antimicrobial sensitivity pattern of extended spectrum $\beta$ lactamases producing E. coli and Klebsiella pneumoniae isolated in a tertiary care hospital. J Medical Sci 2010; 3(4): 265-271.

45. Ullah F, MalikS A, Ahmed J. Antimicrobial susceptibility and ESBL prevalence in Pseudomonas aeruginosa isolated from burn patients in the North West of Pakistan. Burns 2009; 35 (7): 1020-1025. https://doi.org/10.1016/j.burns.2009.01.005

46. Srikumar R, Li XZ, Poole K. Inner membrane efflux components are responsible for $\hat{\mathrm{I}}^{2}$-lactam specificity of multi drug efflux pumps in Pseudomonas aeruginosa. J Bacteriol 1997; 179(2): 7875-7881. https://doi.org/10.1128/jb.179.24.7875-7881.1997

47. Jombo GTA, Jonah P, Ayeni JA. Multidrug Resistant Pseudomonas aeruginosa in Contemporary Medical Practice: Findings from urinary isolates at a Nigerian University teaching hospital. Nigerian J Phys Sci 2008; 23(1-2):105109.https://doi.org/10.4314/njps.v23i1-2.54944

48. Chaudhury A. In vitro activity of Cefpirome: a new fourth generation cephalosporin. Indian J Med Micro 2003; 21 (1): 52-55. PMID: 10992706

49. Strateva T, Ouzounova RV, Markova B, Todorova A, Marteva-ProevskaY, Mitov I. Widespread detection of VEB1-type extended-spectrum beta-lactamases among nosocomial ceftazidime-resistant Pseudomonas aeruginosa isolates in Sofia, Bulgaria. J Chem 2007; 19 (2): 140-145. https://doi.org/10.1179/joc.2007.19.2.140

50. Mathur P, Kapil A, Das B, Dhawan B. Prevalence of extended spectrum $\hat{\mathrm{I}}^{2}$-lactamase producing gram negative bacteria in a tertiary care hospital. Indian J Med Res 2002; 115(2): 153-157. https://doi.org/10.7860/JCDR/2013/6460.3462

51. Nwankwo EOK, Shuaibo SA. Antibiotic susceptibility pattern of clinical isolates of Pseudomonas aeruginosa in a tertiary health institution in Kano, Nigeria J Med Biomed Sci 2010; 37-40. PMID: 22589655

52. Lim KT, Yasin RY, Yeo CC. Genetic fingerprinting and antimicrobial susceptibility profiles of Pseudomonas aeruginosa hospital isolates in Malaysia. J Microbiol Infectious Diseases 2009; 42:197-209. PMID: 19812853

53. Unan D, Gnseren F. The resistance of P. aeruginosa strains isolated from nosocomial infections against various antibiotics. Mikrobiyol Bult. 2000; 34: 255-60 https://doi.org/10.1128/CMR.00040-09

54. Sabir R, Alvi SFD, Fawwad A. Antimicrobial susceptibility pattern of aerobic microbial isolates in a clinical laboratory in Karachi- Pakistan. Pak J Med Sci 2013; 29(3): 851-5 https://doi.org/10.12669/pjms.293.3187

55. Gad GF, El-Domany RA, Zaki S, Ashour HM. Characterization of Pseudomonas aeruginosa Isolated from Clinical and Environmental Samples in Minia, Egypt: Prevalence, Antibiogram and Resistance Mechanisms. J Antimicr Chemother 2007; 60: 1010-7. https://doi.org/10.1093/jac/dkm348

56. Parmar H, Dholakia A, Vasavada D, Singhala H. The current status of antibiotic sensitivity of Pseudomonas aeruginosa isolated from various clinical samples. Int J Res Med 2013; 2(1); 1-6. https://doi.org/10.4103/0253-7613.44156

57. Berglund B. Environmental dissemination of antibiotic resistance genes and correlation to anthropogenic contamination with antibiotics. J Infect Ecol Epidemiol 2015; 5: 28564. https://doi.org/10.3402/iee.v5.28564 